\title{
Differential Psoriatic Effect of Imiquimod on Balb/c and Swiss Mice
}

\author{
Fathima Salwa' Murali Badanthadka² Lidwin D’Souza ${ }^{3}$ \\ ${ }^{1}$ Department of Pharmacology, NGSM Institute of Pharmaceutical \\ Sciences, Mangalore, Karnataka, India \\ ${ }^{2}$ Department of Nitte University Centre for Animal Research and \\ Experimentation, NGSM Institute of Pharmaceutical Sciences \\ (NGSMIPS), Nitte (deemed to be) University, Paneer, Deralakatte, \\ Address for correspondence Murali Badanthadka, MPharm, PhD, \\ Department of Nitte University Centre for Animal Research and \\ Experimentation, NGSM Institute of Pharmaceutical Sciences, \\ Paneer, Deralakatte, Mangalore 575018, Karnataka, India \\ (e-mail: murali@nitte.edu.in).
} Mangalore, Karnataka, India

${ }^{3}$ Department of Pharmacovigilance, Norwich Clinical Services,

Bangalore, Karnataka, India

J Health Allied Sci Nu 2021;11:170-177.

\begin{abstract}
Introduction The influence of animal strain on psoriasis model development by imiquimod (IMQ) has been studied in Balb/c and Swiss mice.

Materials and Methods Female mice of either strain were challenged with 5\% IMQ (62.5 mg on back skin, $10 \mathrm{mg}$ on right ear). They were observed for the severity of the disease using Psoriasis area severity index (PASI), splenomegaly, and histopathological alterations. To validate the model, well-established antipsoriatic drug clobetasol $(0.05 \%, 120 \mathrm{mg}$ on the back skin, $10 \mathrm{mg}$ on the right ear) was used. Additionally, to study the strain-dependent response to IMQ associated with oxidative stress, various antioxidant factors like superoxide dismutase (SOD), catalase (CT), and glutathione (GSH) were measured. Antioxidant natural product curcumin (1\%, $150 \mathrm{mg}$ on back skin, $12.5 \mathrm{mg}$ on right ear) was used to evaluate the alleviation of oxidative stress on distinct mice strain.

Keywords

- Balb/c mice

- clobetasol

- curcumin

- imiquimod

- psoriasis

- Swiss mice

Results PASI score, body weight, and histopathology indicated the development of disease in both the strains, severity, and stability of which was dependent on the particular strain. Splenomegaly suggested the systemic effect, which was comparable in both the stains. IMQ and its involvement in redox status were confirmed by an alteration in the activity of SOD, CT, and levels of GSH.

Conclusion This study demonstrated that, in the IMQ-induced psoriasis model, the genetic background has some impact on the disease severity, stability, and redox imbalance.
\end{abstract}

\section{Introduction}

Psoriasis is an autoimmune, inflammatory, chronic disease characterized by hyperproliferation of epidermal keratinocytes, infiltration of leukocyte, and altered dermal vascularity. It is considered one of the significant causes of health concern affecting $~ 1$ to $2 \%$ of the world population and has more tendencies to occur during early adulthood. The disease develops as a result of a multifactorial condition that is associated with genetic, environmental, and immunological factors and found to be induced by multiple immune dysregulation. ${ }^{1,2}$ published online May 14, 2021
DOI https://doi.org/

$10.1055 / \mathrm{s}-0041-1726681$

ISSN $2582-4287$ (c) 2021. Nitte (Deemed to be University).

This is an open access article published by Thieme under the terms of the Creative Commons Attribution-NonDerivative-NonCommercial-License, permitting copying and reproduction so long as the original work is given appropriate credit. Contents may not be used for commercial purposes, or adapted, remixed, transformed or built upon. (https://creativecommons.org/licenses/by-nc-nd/4.0/).

Thieme Medical and Scientific Publishers Pvt. Ltd. A-12, 2nd Floor, Sector 2, Noida-201301 UP, India 
The complete pathogenesis is still unclear, so the appropriate treatment strategy is not available. While pathogenesis is not fully known, a major challenge in psoriasis research is the lack of an ideal animal model. Research in psoriasis remains challenging because it does not occur naturally in laboratory animals that mirror the disease's phenotype and immunopathogenesis. ${ }^{3}$

Since 2009, activating toll-like receptors (TLR) to induce psoriasis in animals was popularly employed for psoriasis research, because this model offers several advantages, including rapid development, cost-effective, and easy to establish. ${ }^{4}$ Different pathways are involved in the development of imiquimod (IMQ)-induced psoriasis. The various key cytokines like interleukin-17 (IL-17), IL-16, IL-23, IL-35, IL-33, and IL-18 were found to induce or exacerbate psoriatic skin inflammation. ${ }^{4-9}$ The immunological influences like IL-23, IL-12, and tumor necrosis factor- $\alpha(\mathrm{TNF}-\alpha)$ initiate the inflammation process in which IL-12 induces Th-1 driven increase in TNF- $\alpha$ and IL-23. Therefore, the activated cytokines stimulate Th-17 cells, which synthesize proinflammatory mediators like IL-17 and IL-22. The increased concentrations of TNF- $\alpha$, IL-12, and IL-23 have been found clinically in psoriatic skin. ${ }^{10}$

Although this model resembles most psoriasis features, it still has limitations; notably, the immunopathology differs from human psoriasis. As for all mouse models, confounding factors such as mouse strain and sex influence the phenotype. Despite great advances during the last years, psoriasis' immunogenetic characteristic, which has identified several dozens of loci within the genome associated with psoriasis susceptibility, still warrants further research. ${ }^{3}$ Considering the involvement of genetic factors, it is obvious to expect that any realistic mouse model of psoriasis would be sensitive to the genetic background with certain aspects of psoriasis dermatitis phenotype varying among the mouse strains. Focusing on mouse strain, an essential factor affecting disease development, we aimed to assess disease severity and stability.

Recent studies suggest that oxidative stress may contribute the psoriasis pathogenesis. The antioxidant system plays a major role in maintaining lipid peroxidation, deoxyribonucleic acid (DNA) modification, and inflammatory molecule secretion. When this system is compromised, it leads to oxidative stress. ${ }^{11,12}$

The various mechanisms like the capacity of DCs to promote T-cell activation, proliferation, and pro-inflammatory cytokine production, TLR mediated reactive oxygen species (ROS) production tends to regulate the T-cell activation. Further, deficient counter-regulatory response offered by oxidative stress on Th2 to Th1 for psoriasis, ROS involvement in the activation of transcription factors in Kupfer cells leading to hyperproliferation. ROS induced angiogenic process through vascular endothelial growth factor (VEGF)dependent and VEGF independent pathways and ROS role as secondary messengers that modulate transcription factors indifferent inflammatory signaling pathways like mitogen-activated protein kinase (MAPK), NF-KB (nuclear factor Kappa light chain enhancer of activated B cells), and
Janus kinase-signal transducers and activators of transcription (JAK-STAT) involved in the oxidative stress-dependent disease development. ${ }^{13-17}$ As IMQ induces psoriasis in a strain-dependent manner, we made an attempt to know the role of animal strain on psoriasis associated with oxidative stress. The natural antioxidant product curcumin was used to evaluate its effect on alleviating oxidative stress medicated psoriasis induced by IMQ.

\section{Materials and Methods}

\section{Animals}

Eight to twelve weeks old healthy female Swiss mice and Balb/c mice weighing around 20 to $25 \mathrm{~g}$ were obtained from NUCARE (Nitte University Centre for Animal Research and Experimentation), Deralakatte, Mangalore. Animals were maintained under standard laboratory conditions of a temperature $22 \pm 2^{\circ} \mathrm{C}$ with 12 hours light-dark cycle. Food and water were provided ad libitum. As per the guidelines of CPCSEA (Committee for the Purpose of Control and Supervision of Experiment on Animals), New Delhi, and IAEC (Institutional Animal Ethics Committee), all the animal experiments were performed.

\section{IMQ-Induced Psoriasis Mouse Model}

The animal's dorsal skin was shaved 2 days before applying drugs using Veet hair removal cream. Further, animals were divided into four different groups $(n=6)$ groups: Group I, Vaseline control; Group II, IMQ control; Group III, IMQ + 0.05\% of clobetasol group; and Group IV, IMQ + 1\% curcumin gel group. Psoriasis was induced as described by van der Fits. ${ }^{4}$ In brief, $62.5 \mathrm{mg}$ of $5 \%$ IMQ was applied topically on shaved dorsal skin and $5 \mathrm{mg}$ on mice's right ear for 6 consecutive days. Normal control was treated similarly with $62.5 \mathrm{mg}$ Vaseline and $5 \mathrm{mg}$ on the right ear. Group III and IV were treated with $0.05 \%$ clobetasol cream (120mg on back skin, 10mg on right ear), and $1 \%$ curcumin gel (120mg on back and $12.5 \mathrm{mg}$ on right ear) respectively after four hours of application with IMQ. The diagram representing the experimental design is shown in $\boldsymbol{- F i g} \mathbf{1}$.

\section{Scoring the Severity of the Disease}

To score the severity of inflammation in the back skin, Psoriasis area severity index (PASI) scoring system was used as described by van der Fits et al. ${ }^{4}$ Erythema, scaling, and thickening were scored independently on a scale from 0 to 4 (0-none, 1-slight, 2-moderate, 3-marked, 4-very marked). The combination of the above independent score is considered a cumulative score for PASI, which served as a measure of psoriasis severity. Ear thickness and back skin thickness were measured using a digital caliper (Aerospace, Maroor's Machine Tools Co., Mangalore, India). Body weight and food intake were recorded daily. ${ }^{5}$

\section{Evaluation of the Systemic Effect of IMQ on the Spleen}

After 24 hours of the final application of IMQ the animals were euthanized by isoflurane inhalation. The spleen was excised and weighed to calculate the spleen index (spleen index $=$ spleen weight $/$ body weight . 


\section{Experimental design}

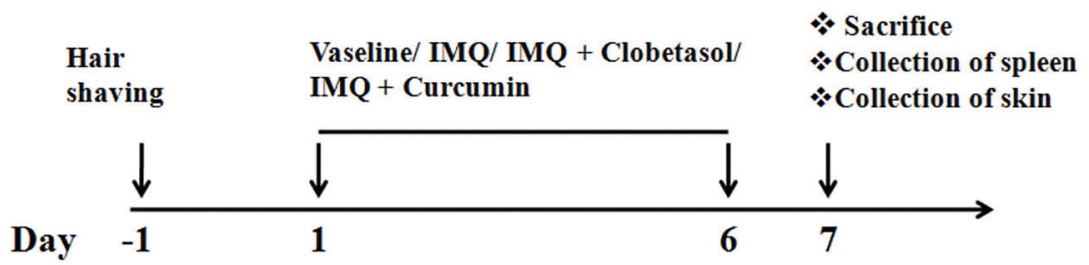

Groups and Treatment:

Group- I: Vaseline control (Topical application of $62.5 \mathrm{mg}$ of vaseline on back and $5 \mathrm{mg}$ to right ear) Group - II: IMQ control (Topical application of $62.5 \mathrm{mg} 5 \%$ IMQ on back and $5 \mathrm{mg}$ to right ear) Group-III: IMQ + Clobetasol (Topical application of $62.5 \mathrm{mg} 5 \%$ IMQ on back and $5 \mathrm{mg}$ to right ear. After four hours, topical application of $0.05 \%$ clobetasol $120 \mathrm{mg}$ on back and $10 \mathrm{mg}$ on right ear) Group-IV: IMQ + Curcumin (Topical application of $62.5 \mathrm{mg} \mathrm{5 \%} \mathrm{IMQ} \mathrm{on} \mathrm{back} \mathrm{and} 5 \mathrm{mg}$ to right ear. After four hours, topical application of $1 \%$ curcumin $150 \mathrm{mg}$ on back and $12.5 \mathrm{mg}$ on right ear)

Fig. 1 Experimental design comprising animal groups and the evaluation of different parameters.

\section{Biochemical Evaluation}

The dorsal skin samples were homogenized and used to evaluate oxidative stress in the skin. Five percent of the tissue homogenate was prepared using ice-cold phosphate buffer (0.25M, pH7.4). It was centrifuged at $10,000 \mathrm{rpm}$ for 20 minutes. The supernatant was collected and filtered. The filtrate was used for the estimation of glutathione (GSH), ${ }^{18}$ superoxide dismutase (SOD), ${ }^{19}$ catalase (CT),${ }^{20}$ and total protein content. ${ }^{21}$

\section{Histopathology Studies}

The dorsal skin samples were removed and preserved in 10\% formalin and stored for histopathology studies. Sections were stained using hematoxylin and eosin staining. Analysis of histopathological alteration for psoriasis was done using Baker's scoring system.22

\section{Statistical Analysis}

The results were expressed as mean \pm SEM, with $n=6$ in each group. The results were analyzed by one way analysis of variance) followed by Newman-Keuls test for multiple comparisons for determining the statistical significance between different groups was done by using Graph Pad Prism, version 5 software. $p$-Value $<0.05$ was considered as significant $\left({ }^{*} p<\right.$ $0.05,{ }^{* *} p<0.01,{ }^{* * *} p<0.001$ ).

\section{Results}

\section{The Severity of the Disease}

PASI scoring was done to evaluate the severity of psoriasis daily. Signs of psoriasis were not seen in Vaseline control (Group I). The PASI score was increased in Group II from day 2 and significantly higher $(p<0.001)$ severity on day 6 , followed by a decline in severity on day 7 in both the strains of mice. These results indicate the successful development of the disease. Treatment with clobetasol (Group III) or curcumin (Group IV) decreased the overall skin lesions significantly in both the strains ( $\mathbf{- F i g .} \mathbf{2 A}$ and $\mathbf{B}$ ).

Vaseline treatment did not alter the animal's body weight, suggesting no adverse effect of Vaseline on the animal's body weight. However, IMQ application significantly decreased the animal's body weight, meaning the diseased state of animals (6-13\% in Swiss mice, 3-8\% in case of Balb/c mice) as early as day 3 and reversed thereafter. However, both clobetasol and curcumin applications did not reverse body weight of Swiss mice. However, in Balb/c mice, the reversal in body weight was seen only in the curcumin-treated group ( - Fig. 2 C and $\mathbf{D}$ ). We found an increased ear thickness of IMQ-treated animals without statistical significance (data not shown for simplicity).

\section{Effect of IMQ on the Spleen}

IMQ application on the shaved dorsal skin of mice for 6 consecutive days led to a significant increase in the spleen index in Group II compared with group I $(p<0.01$ Swiss mice, $p<0.001 \mathrm{Balb} / \mathrm{c}$ mice). Treatment with clobetasol significantly reduced spleen index in both the strains $(p<0.001)$. The overall result suggested that IMQ affects the spleen, and it induces splenomegaly, which can be reduced by clobetasol treatment. There was no protective effect in curcumin-treated animals from splenomegaly.

\section{Histopathology Studies}

The study was done for histopathological changes in control groups: IMQ-induced psoriasis groups without treatment and IMQ-induced psoriasis groups with the treatment comprising clobetasol and curcumin. This was done in two strains of mice: Swiss albino mice and Balb/c mice. To assess the disease severity at the histopathological level, Baker's scoring system was used.

In the Vaseline group, total Baker's score was 2.66 in both the mice's strain. In the IMQ-treated group, Baker's score increased to 4.5 in Swiss mice and 3.33 in Balb/c mice. 
A
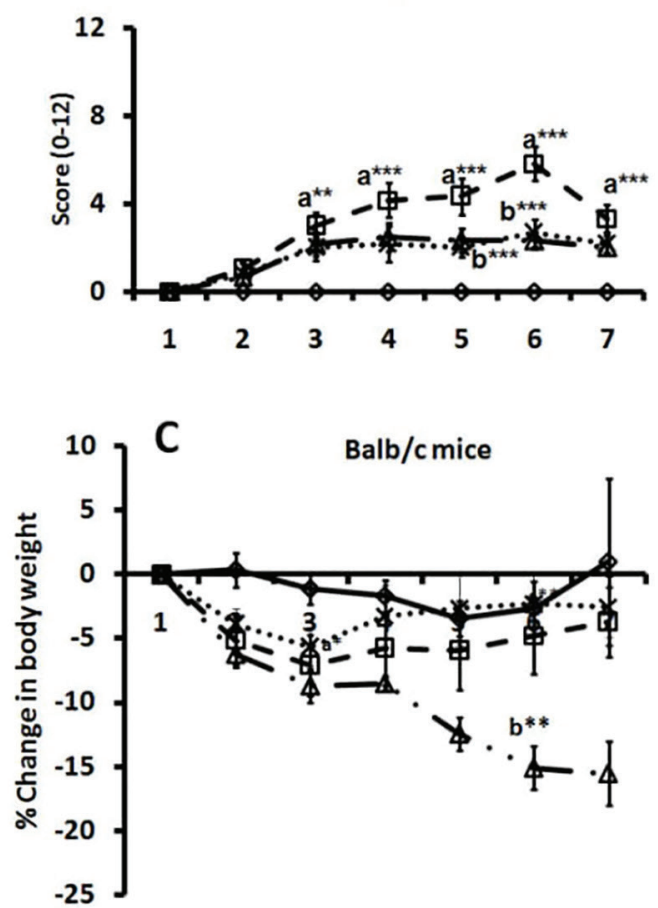

E

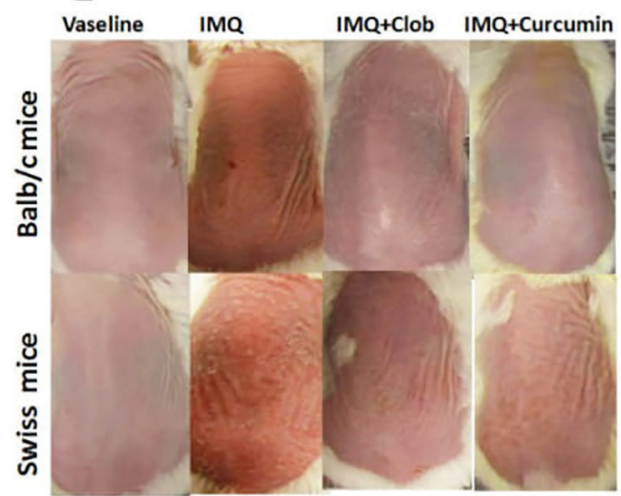

B
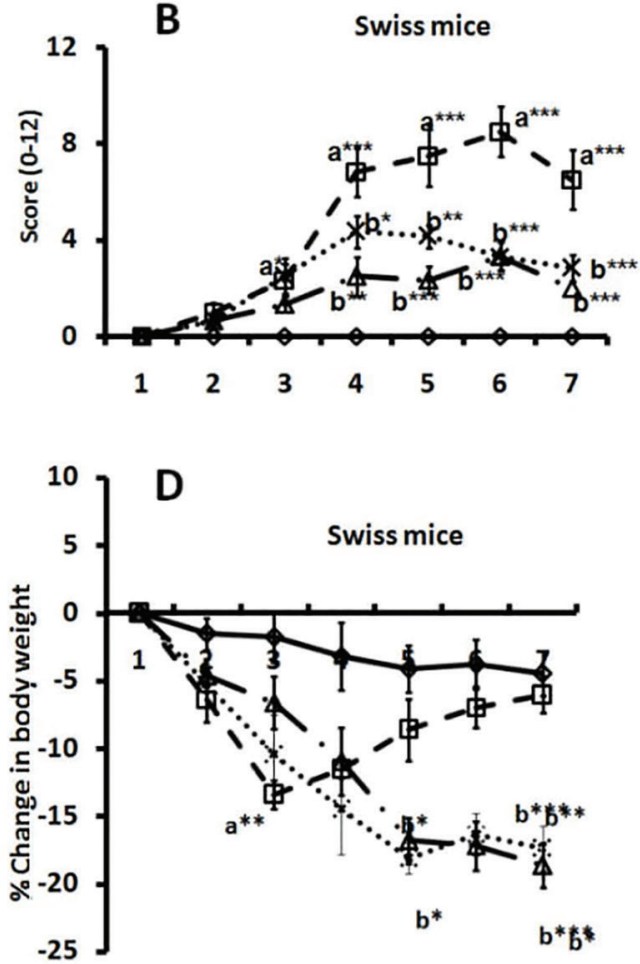

$F$

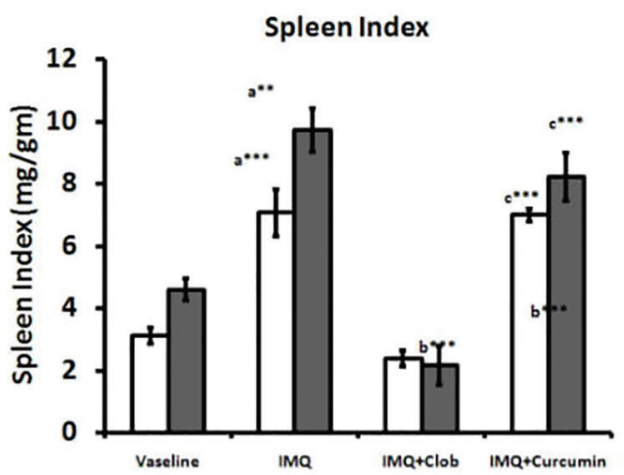

$ه$ Vaseline

๑ IMQ

$\triangle \mathrm{IMQ}+\mathrm{Clob}$

. IMQ+Curcumin

口Balb/c 口Swiss

Fig. 2 Evaluation of local and systematic development of the disease. Psoriasis was developed in the mice by the topical application of imiquimod (IMQ). Disease severity was measured using Psoriasis area severity index (PASI) scoring system that is the cumulative score of individual score of erythema, scaling, and thickness. The cumulative score (PASI)(A and $\mathbf{B})$. The effect of drugs on the body weight of mice represented as \% change in body weight (C, D). The representative picture showing development of disease by IMQ and attenuation by clobetasol (Clob) or curcumin (E). The systematic effect of IMQ was assessed by measuring the Spleen index (F). All values are expressed as mean \pm standard error of mean. a: compared against Vaseline, b: compared against IMQ, c: compared against IMQ +Clob. Comparison was done with respective strain $\left({ }^{*} p<0.05,{ }^{* *} p<0.01,{ }^{* * *} p<0.001\right)$.

There was a significant increase in Baker's score in both the strains compared with the Vaseline control group ( $p<$ 0.001 Swiss mice, $p<0.01 \mathrm{Balb} / \mathrm{c}$ mice). In clobetasol-treated group, Baker's scoring was significantly reduced to 3 ( $p$ $<0.001)$ in Swiss mice and $2.5(p<0.01)$ in Balb/c mice. In the curcumin-treated group, Baker's score was significantly reduced to $3.5(p<0.001)$ in Swiss mice and 3 in Balb/c mice (-Fig. 3).

Oxidative Status in IMQ-Induced Psoriasis Skin in Mice IMQ induced psoriatic mice to have lower SOD levels, CT, and GSH, and higher levels of protein content than the Vaseline treated group ( - Fig. 4). Results of Swiss mice showed a significant decrease in SOD and catalase activities and a significant increase in protein content $(p<0.001)$ compared with the Vaseline group. However, treatment with clobetasol increased activities of SOD, CT, and GSH. Similarly, SOD and CT elevation were seen in the curcumin-treated group ( $p<0.001, p<0.05$ ). When compared between clobetasol and curcumin-treated groups, curcumin showed a significant increase in CT activity ( $p<0.05$ ). Results of Balb/c mice showed a significant decrease in SOD and GSH levels and a significant increase in protein content $(p<0.001)$ in IMQ-treated group compared with the Vaseline group. Treatment with clobetasol and curcumin showed a significant elevation of SOD $(p<0.05)$ and GSH $(p<0.001)$ levels. 
A



B

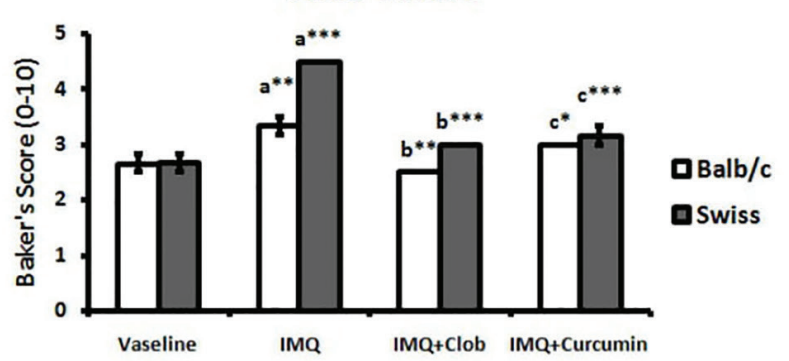

Fig. 3 Histopathological view of dorsal skin of mice (A). Pathological disease severity by histopathology is represented as Baker's score (B). All values are expressed as mean \pm standard error of mean. a: compared against Vaseline, b: compared against imiquimod (IMQ), c: compared against IMQ + clobetasol. The comparison was done with respective strain $\left({ }^{*} p<0.05,{ }^{* *} p<0.01,{ }^{* * *} p<0.001\right)$.
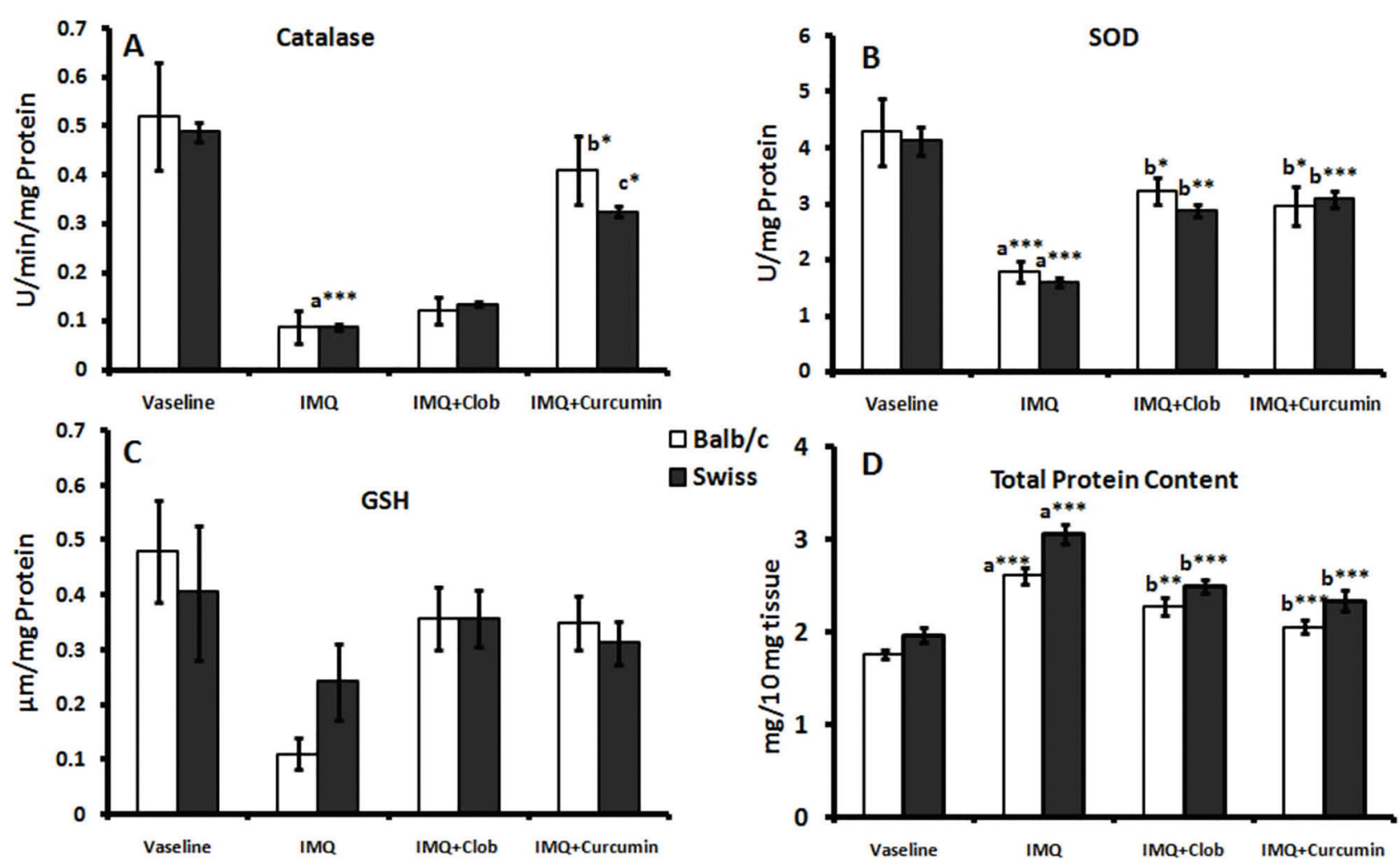

Fig. 4 Oxidative stress evaluated in skin. The $5 \%$ of tissue homogenate was prepared and subjected for estimation of catalase (A), superoxide dismutase (SOD) (B), glutathione (GSH) (C), and total protein content (D). All values are expressed as mean \pm standard error of mean. a: compared against Vaseline, b: compared against imiquimod (IMQ), c: compared against IMQ + clobetasol. The comparison was done with respective strain $\left({ }^{*} p<0.05,{ }^{* *} p<0.01,{ }^{* * *} p<0.001\right)$.

The overall result suggested that IMQ treatment causes oxidative stress, which can be restored by treatment with clobetasol or curcumin in both the strains.

\section{Discussion}

Animal models represent complex systems, and it is essential to explain the genesis and disease progression. These models are used to develop new therapeutic agents, and they play a critical role in building the translational gap in clinical research. The selection of validated and extrapolative animal models is very crucial to report clinical questions. ${ }^{23}$ Psoriasis is a multifactorial, complex autoimmune disease. Mechanisms of the disease are highly complicated, involving several key molecules, such as cytokine keratinocytes, inflammatory cells (CD4+ and CD8+ $T$ cells, various types of dendritic cells, neutrophils, and natural killer T cells and mast cells), and growth factors. The involvement of keratinocytes in the inflammation process is via surface cytokine receptors. The stimulated abnormal immune response leads to keratinocyte hyperproliferation. To understand the disease's complexity, an animal model representing all feature of the disease 
is necessary. The current therapeutic strategy can manage the disease but does not ensure the complete cure. ${ }^{24}$ So far no one model can meet all of the research needs for psoriasis. Therefore, the choice of model system will depend on the questions being addressed. ${ }^{25}$ Lack of appropriate animal models that mimic skin disease characteristics cause significant difficulty in understanding psoriasis pathogenesis and new drug development. ${ }^{26}$ Therefore, the development of a stable model is essential.

IMQ induced cutaneous phenotype in mice, which closely resembles human psoriasis. Considering its advantages, the utilization of this model had significantly increased. Various products are screened for their antipsoriatic effects by using this model. Several immunological pathways and cytokines involved in the disease's genesis and progression were discovered using this model..$^{27}$ Despite its advantages, this model still suffers from significant drawbacks, one of which includes its dependency on the animal strain. Flutter and Nestle summarized the strain-dependent effect of various mice, including Balb/c mice ${ }^{5}$ that developed disease more rapidly and causes more flaky and inflamed skin than in C57bl/6 mice. Similarly, in 129/Sv mice, parakeratosis and epidermal hyperplasia are more marked than in $\mathrm{C} 57 \mathrm{bl} / 6$ mice. In contrast to the reduced responses in the skin, the systemic side effects are more severe on the C57bl/ 6 background, where mice suffer dehydration (recoverable by injection of PBS) and fever secondary to high serum levels IL-6. ${ }^{5}$ Swindell et al concluded that IMQ-mediated model is not the unique model of psoriasis but triggers a core set of active pathways in diverse skin diseases. ${ }^{28}$ This model represents the first phase of the human biphasic psoriasis disease characterized by plaque-type psoriatic lesions dominated by neutrophil infiltration before the late stage with chronic plaques associated with Th1 response. Balb/c develops an immune response, mainly Th2-dependent. ${ }^{29}$

It was evident from the literature search that $\mathrm{Balb} / \mathrm{c}$ and C57bl/6 are the two most widely used mice strains for psoriasis. However, Swiss albino mice are not used or rarely used. As of now, we could find only a few studies done using the Swiss model. ${ }^{30,31}$ Therefore, in the present study, we developed a psoriasis model in Swiss albino mice and Balb/c mice and compared the disease stability and severity. Clobetasol is used as a standard drug to validate the models.

Topical treatment of IMQ on the shaved dorsal skin of mice and right ear leads to increased PASI score and ear thickness of mice due to hyperproliferation, epidermal hyperkeratosis, and increase in cytokine release that resulted in erythema and epidermal thickness. The epidermal hyperplasia can be correlated with the appearance of STAT3 that is a key transcription activator involved in the development of psoriatic lesions. ${ }^{32}$ By looking into PASI in both the strains, we found that Swiss mice have more severe psoriasis lesion than the Balb/c mice. As per our knowledge, this study is the first to report results based on a comparison of IMQ responses between Swiss mice and Balb/C mice.

Weight loss and anorexia are also the symptoms of generalized pustular psoriasis, and these phenotypes are dependent on IL-1 and IL-36. ${ }^{33}$ To check the health status of mice, body weight and food intake were measured daily. In our study, we found mild weight loss in IMQ treated groups and also in the curcumin group, whereas the body weight of the clobetasol treated group showed a consistent decrease in both the strains suggesting clobetasol-mediated toxicity in both the strains.

Several studies have been reported splenomegaly in IMQ-induced psoriasis. ${ }^{28,34,35}$ Spleen is one of the crucial organs involved in both innate and adaptive immune systems. It is considered to be the most significant filter of blood in the body. ${ }^{36}$ In our study, we found splenomegaly in IMQ-treated groups of both the strains, suggesting that topical application of IMQ also shows the systemic effect that may indicate an increased inflammatory immune response. This effect was comparable in both the strains. Cotreatment of IMQ with clobetasol significantly reduces the spleen's size and weight below average in both the strains. Clobetasol is a corticosteroid used in the treatment of psoriasis, and their systemic effect varies in different tissues, which are mostly influenced by the intrinsic activity of the drug molecule and route of administration. ${ }^{37}$ There was a reduction in spleen size in the curcumin group of Swiss mice compared with the IMQ group indicating the curcumin could protect mice from IMQ-induced splenomegaly, specifically in Swiss mice. It was reported that curcumin is the most effective treatment for psoriasis by inhibiting cytokines such as IL-17A, IL-17F, and other inflammatory factors (TNF- $\alpha$ ). In clinical observations, lower PASI scores proved it effectiveness. ${ }^{38}$ In Balb/c mice, curcumin did not provide any protective effect due to the systemic side-effect of IMQ. These results are due to the strain-specific response of mice to chemical stimuli. Studies suggest many variances between different animal species and even within the same specie's strain individuals around life span. ${ }^{39}$ Earlier reports indicated that Balb/c immunized with cruzipain-induced heart autoimmunity, whereas C57bl/6 mice were resistant. Therefore, during the immune response, different genetic background affects several parameters like B cell activation. ${ }^{40}$

Skin is continuously exposed to ultraviolet radiation, other chemicals, and environmental stress that lead to ROS. Thus, the skin is considered to be the primary target for oxidative damage. ${ }^{11}$ However, endogenous antioxidant enzymes like CT, SOD, and GSH peroxidase will balance ROS levels and inhibit ROS-induced damage and act as a defense system. But when ROS are present in more number for a long time, the endogenous antioxidant present will not be sufficient to fight against the ROS present, which results in cellular-mediated inflammation and develops various skin disorders that include psoriasis. ${ }^{37}$

Psoriasis is found to be associated with increased ROS production and a reduction in antioxidants. ROS-induced damage causes lipid peroxidation, modification of DNA, and increased release of inflammatory cytokines. Oxidative damage results in T-cells and keratinocytes' activation and is also responsible for releasing pro-inflammatory cytokines, thus triggering inflammatory responses in psoriasis. ${ }^{41}$ ROS act as a second messenger influencing proinflammatory signaling pathways, MAPK/activator 
protein-1 (MAPK/AP-1), JAK-STAT, and NF-кB, and activation of these cellular signal transduction process activates cytokines and neurotransmitters that leads to hyperproliferation, irregular differentiation of cells that is seen in psoriasis. ${ }^{11,42}$ SOD is considered the most crucial antioxidant enzyme system required to convert superoxide anion into hydrogen peroxide and molecular oxygen. In several earlier studies, reports were saying that there is a reduction in SOD activity in IMQ-induced psoriasis skin lesion because of increased superoxide anion production and decreased CT in IMQ-induced psoriasis skin. ${ }^{37}$ In our present study, there was a significant decrease in SOD, CT activity, and GSH levels compared with Vaseline group. Total protein content in the skin homogenate of IMQ group was found to be increased. This may be because of hyperkeratinization of the epidermis, which is a significant feature of psoriasis. There was an increase in SOD, CT activity, and GSH levels in clobetasol and curcumin groups in both the strains compared with the disease control IMQ group. Curcumin group has a more significant effect on Swiss mice, indicating a strain-dependent effect. Curcumin's antioxidant property reduces oxidative stress, which prevents oxidative damage and may promote early recovery.

The psoriasis research is challenging because of the lack of an ideal animal model with IMQ-induced psoriasis that is considered most effective. Although this model offers certain advantages, it still has significant disadvantages. The model's stability and severity are dependent on animal strain. The interpretation of particular strain results should be made with great caution since distinct strains differ in specific characteristics. While Swiss mice are rarely used for psoriasis study, Balb/c mice are more frequently utilized. From the present study, we found that Swiss mice provide a better psoriatic model as compared with Balb/c in terms of both severity and disease stability.

\section{Conclusion}

Our work gives an idea about disease severity and stability of IMQ-induced psoriasis in two distinct animal strains. Application of IMQ induces psoriasis in Balb/c and Swiss mice in a strain-dependent manner. The Swiss model shows more severe disease as compared with Balb/c model. Further, Swiss model was found to be better both in terms of disease stability and severity.

\section{Conflict of Interest \\ None declared.}

\section{Acknowledgment}

We thank Dr. Hrishikesh Damle, MD and CEO, Atrimed Pharmaceutical Pvt Ltd, Bangalore, for preparing curcumin gel for our study.

\section{References}

1 Alwan W, Nestle FO. Pathogenesis and treatment of psoriasis: exploiting pathophysiological pathways for precision medicine. Clin Exp Rheumatol 2015;33(5, Suppl 93):S2-S6
2 Krueger JG, Bowcock A. Psoriasis pathophysiology: current concepts of pathogenesis. Ann Rheum Dis 2005;64(Suppl 2): ii30-ii36

3 Schön MP, Manzke V, Erpenbeck L. Animal models of psoriasis-highlights and drawbacks. J Allergy Clin Immunol 2021;147(2):439-455

4 van der Fits L, Mourits S, Voerman JS, et al. Imiquimod-induced psoriasis-like skin inflammation in mice is mediated via the IL-23/IL-17 axis. J Immunol 2009;182(9):5836-5845

5 Flutter B, Nestle FO. TLRs to cytokines: mechanistic insights from the imiquimod mouse model of psoriasis. Eur J Immunol 2013;43(12):3138-3146

6 Yoshiki R, Kabashima K, Honda T, et al. IL-23 from Langerhans cells is required for the development of imiquimod-induced psoriasis-like dermatitis by induction of IL-17A-producing! $[$ T cells. J Invest Dermatol 2014;134(7):1912-1921

7 Zhang J, Lin Y, Li C, et al. IL-35 decelerates the inflammatory process by regulating inflammatory cytokine secretion and M1/M2 macrophage ratio in psoriasis. J Immunol 2016; 197(6):2131-2144

8 Duan Y, Dong Y, Hu H, et al. IL-33 contributes to disease severity in psoriasis-like models of mouse. Cytokine 2019;119:159-167

9 Niu XL, Huang Y, Gao YL, et al. Interleukin-18 exacerbates skin inflammation and affects microabscesses and scale formation in a mouse model of imiquimod-induced psoriasis. Chin Med J (Engl) 2019;132(6):690-698

10 Weger W. Current status and new developments in the treatment of psoriasis and psoriatic arthritis with biological agents. Br J Pharmacol 2010;160(4):810-820

11 Kadam DP, Suryakar AN, Ankush RD, Kadam CY, Deshpande KH. Role of oxidative stress in various stages of psoriasis. Indian J Clin Biochem 2010;25(4):388-392

12 Sunkari S, Thatikonda S, Pooladanda V, Challa VS, Godugu C. Protective effects of ambroxol in psoriasis like skin inflammation: exploration of possible mechanisms. Int Immunopharmacol 2019;71:301-312

13 Lin X, Huang T. Oxidative stress in psoriasis and potential therapeutic use of antioxidants. Free Radic Res 2016;50(6):585-595

14 Gabr SA, Al-Ghadir AH. Role of cellular oxidative stress and cytochrome $\mathrm{c}$ in the pathogenesis of psoriasis. Arch Dermatol Res 2012;304(6):451-457

15 Yin LL, Zhang Y, Guo DM, An K, Yin MS, Cui X. Effects of zinc on interleukins and antioxidant enzyme values in psoriasis-induced mice. Biol Trace Elem Res 2013; 155(3):411-415

16 Birben E, Sahiner UM, Sackesen C, Erzurum S, Kalayci O. Oxidative stress and antioxidant defense. World Allergy Organ J 2012;5(1):9-19

17 Kaur M, Sharma S, Kukreja S, et al. Study of oxidative stress in patients of psoriasis. Int J Res Dermatol. 2016;2(4):95-98

18 Ellman GL. Tissue sulfhydryl groups. Arch Biochem Biophys 1959;82(1):70-77

19 Kakkar P, Das B, Viswanathan PN. A modified spectrophotometric assay of superoxide dismutase. Indian J Biochem Biophys 1984;21(2):130-132

20 Aebi H. Catalase in vitro. Methods Enzymol 1984;105:121-126

21 Lowry OH, Rosebrough NJ, Farr AL, Randall RJ. Protein measurement with the Folin phenol reagent. J Biol Chem 1951;193(1):265-275

22 Baker BS, Brent L, Valdimarsson H, et al. Is epidermal cell proliferation in psoriatic skin grafts on nude mice driven by T-cell derived cytokines? Br J Dermatol 1992;126(2):105-110

23 Denayer T, Stöhr T, Roy MV. Animal models in translational medicine: validation and prediction. New Horiz Transl Med 2014;2:5-11

24 Niculet E, Radaschin DS, Nastase F, et al. Influence of phytochemicals in induced psoriasis (Review). Exp Ther Med 2020;20(4):3421-3424 
25 Bocheńska K, Smolińska E, Moskot M, Jakóbkiewicz-Banecka J, Gabig-Cimińska M. Models in the research process of psoriasis. Int J Mol Sci 2017;18(12):2514

26 Di Domizio J, Conrad C, Gilliet M. Xenotransplantation model of Psoriasis. Methods Mol Biol 2017;1559:83-90

27 Badanthadka M, D'Souza L. Imiquimod induced psoriasis mice model: a promising tool for psoriasis research? Res J Pharm Tech. 2020;13(7):3508-3515

28 Swindell WR, Michaels KA, Sutter AJ, et al. Imiquimod has strain-dependent effects in mice and does not uniquely model human psoriasis. Genome Med 2017;9(1):24

29 Terhorst D, Chelbi R, Wohn C, et al. Dynamics and transcriptomics of skin dendritic cells and macrophages in an imiquimod-induced, biphasic mouse model of psoriasis. J Immunol 2015;195(10):4953-4961

30 Parmar KM, Itankar PR, Joshi A, Prasad SK. Anti-psoriatic potential of Solanum xanthocarpum stem in imiquimod-induced psoriatic mice model. J Ethnopharmacol 2017;198:158-166

31 Pukale SS, Sharma S, Dalela M, et al. Multi-component clobetasol-loaded monolithic lipid-polymer hybrid nanoparticles ameliorate imiquimod-induced psoriasis-like skin inflammation in Swiss albino mice. Acta Biomater 2020;115:393-409

32 Sun S, Zhang X, Xu M, et al. Berberine downregulates CDC6 and inhibits proliferation via targeting JAK-STAT3 signaling in keratinocytes. Cell Death Dis 2019;10(4):274

33 Alvarez P, Jensen LE. Imiquimod treatment causes systemic disease in mice resembling generalized pustular psoriasis in an IL-1 and IL-36 dependent manner. Mediators Inflamm 2016;2016:6756138
34 Chen $\mathrm{HH}$, Chao YH, Chen DY, et al. Oral administration of acarbose ameliorates imiquimod-induced psoriasis-like dermatitis in a mouse model. Int Immunopharmacol 2016;33:70-82

35 Zhang S, Liu X, Mei L, Wang H, Fang F. Epigallocatechin-3-gallate (EGCG) inhibits imiquimod-induced psoriasis-like inflammation of BALB/c mice. BMC Complement Altern Med 2016; 16(1):334

36 Mebius RE, Kraal G. Structure and function of the spleen. Nat Rev Immunol 2005;5(8):606-616

37 Zhou Q Mrowietz U, Rostami-Yazdi M. Oxidative stress in the pathogenesis of psoriasis. Free Radic Biol Med 2009;47(7):891-905

38 Hosseini A, Hosseinzadeh $\mathrm{H}$. Antidotal or protective effects of Curcuma longa (turmeric) and its active ingredient, curcumin, against natural and chemical toxicities: a review. Biomed Pharmacother 2018;99:411-421

39 Al-Zahaby SA, Salem ML, Abdul-Aziz3 KK, et al. Comparative experimental studies on the spleen of young and aged balb/c and cd-1 mice. Int J Adv Res Biol Sci. 2017;4(12):226-241

40 Pellegrini A, Guiñazú N, Aoki MP, et al. Spleen B cells from $\mathrm{BALB} / \mathrm{C}$ are more prone to activation than spleen $\mathrm{B}$ cells from C57BL/6 mice during a secondary immune response to cruzipain. Int Immunol 2007;19(12):1395-1402

41 Xu F, Xu J, Xiong X, Deng Y. Salidroside inhibits MAPK, NF-кB, and STAT3 pathways in psoriasis-associated oxidative stress via SIRT1 activation. Redox Rep 2019;24(1):70-74

42 Baek JO, Byamba D, Wu WH, Kim TG, Lee MG. Assessment of an imiquimod-induced psoriatic mouse model in relation to oxidative stress. Arch Dermatol Res 2012;304(9):699-706 